\title{
Specific organ metastases and survival in metastatic non-small-cell lung cancer
}

\author{
TOMOHIRO TAMURA ${ }^{1}$, KOICHI KURISHIMA ${ }^{2}$, KENSUKE NAKAZAWA $^{1}$, KATSUNORI KAGOHASHI ${ }^{3}$, \\ HIROICHI ISHIKAWA ${ }^{2}$, HIROAKI SATOH ${ }^{3}$ and NOBUYUKI HIZAWA ${ }^{1}$ \\ ${ }^{1}$ Division of Respiratory Medicine, Institute of Clinical Medicine, University of Tsukuba; \\ ${ }^{2}$ Division of Respiratory Medicine, Tsukuba Medical Center Hospital; ${ }^{3}$ Division of Respiratory Medicine, \\ Mito Medical Center, University of Tsukuba, Mito, Ibaraki, Japan
}

Received July 25, 2014; Accepted August 27, 2014

DOI: $10.3892 / \operatorname{mco} .2014 .410$

\begin{abstract}
The present retrospective study was performed to evaluate the clinicopathological characteristics associated with distant metastasis from non-small-cell lung cancer (NSCLC). The records of NSCLC patients with metastasis at the time of diagnosis between 1999 and 2012 were reviewed. Of the consecutive 1,542 NSCLC patients diagnosed during the study period, 729 (47.3\%) patients presented with distant metastasis. Among those 729 metastatic NSCLC patients, 250 (34.3\%), 234 (32.1\%), 207 (28.4\%), 122 (16.7\%), 98 (13.4\%) and $69(9.5 \%)$ had bone, lung, brain, adrenal gland, liver and extrathoracic lymph node metastasis, respectively. In a multivariate analysis using the Cox proportional hazards model, liver and adrenal gland metastases were unfavorable prognostic factors. However, brain and bone metastases were not statistically significant prognostic factors. Using a logistic regression analysis, metastasis to the adrenal glands and the presence of pleural and/or pericardial fluid effusion were correlated with a poor performance status. Therefore, when planning the treatment of NSCLC patients, particularly those with liver and adrenal gland metastases, we should take into consideration information regarding these unfavorable organ metastases.
\end{abstract}

\section{Introduction}

Distant metastases at the time of presentation of non-small-cell lung cancer (NSCLC) are a frequent clinical problem. Approximately 30-40\% of NSCLC patients present with metastatic disease at the time of diagnosis $(1,2)$. The most common metastatic site is bone, followed by the lungs, brain, liver and adrenal glands. Clinically, treatment for metastatic NSCLC consists only of systemic therapy using cytotoxic

Correspondence to: Professor Hiroaki Satoh, Division of Respiratory Medicine, Mito Medical Center, University of Tsukuba, Miya-machi 3-2-7, Mito, Ibaraki 310-0015, Japan

E-mail: hirosato@md.tsukuba.ac.jp

Key words: non-small-cell lung cancer, metastasis, survival and/or molecularly-targeted agents and palliative radiotherapy for symptomatic metastases. It is generally considered that the life expectancy of NSCLC patients depends on the extent of the disease and the response to chemotherapy. Performance status (PS), gender and weight loss have been evaluated as prognostic factors (3). Specific organ metastasis also appears to exert an effect on survival, although this has not been clearly determined (4-6). In this study, using our database of NSCLC patients, we investigated whether specific organ metastasis at the time of presentation was of prognostic significance in NSCLC patients.

\section{Materials and methods}

Patient staging. All the consecutive patients who were diagnosed with metastatic NSCLC in our Divisions of Respiratory Medicine of the University of Tsukuba Hospital and Tsukuba Medical Center Hospital between 1999 and 2012 were retrospectively analyzed. In all the patients, the diagnosis of NSCLC was confirmed with pathological and/or cytological specimens. The pathological diagnosis of NSCLC was determined according to the World Health Organization classification (7). A staging procedure was performed for all the patients prior to any treatment, using head computed tomography (CT) or magnetic resonance imaging (MRI), bone scintigraphy, as well as ultrasonography and/or abdominal CT. The patients were staged according to the Union for International Cancer Control (7th edition) TNM classification (8).

Metastatic locations. We evaluated the clinical significance of each organ metastasis for survival. In addition, in order to evaluate the clinical significance of metastases to different body regions, the metastases were divided according to region, namely brain/head, thorax, abdomen and extremities. Brain/head metastases included those in the brain, eyes, nasal sinuses and gingiva. Thoracic metastases included the lungs, pleura, pericardium and accumulation of pleural/pericardial fluid. Abdominal metastases included the liver, adrenal glands, pancreas, spleen, kidneys, gastrointestinal tract, peritoneum and abdominal lymph nodes. Finally, metastases in the extremities included muscle, bone, bone marrow and skin metastases. 
Statistical analysis. The Kaplan-Meier method was used to assess survival curves and the log-rank test was used in the univariate analysis to evaluate the statistical significance of survival between two groups. The survival time was defined as the interval (in months) between the date of initial therapy or supportive care until the date of death or the date of the last follow-up. Significant variables identified in the univariate analysis were included in the multivariate survival analysis using the Cox proportional hazards model to investigate the effects of clinicopathological factors on survival (9). Logistic regression analysis was applied in order to determine whether the metastatic location was associated with PS and the response to chemotherapy. All the statistical analyses were performed using StatView software for Windows, version 5.0 (SAS Institute Inc., Cary, NC, USA) and $\mathrm{P}<0.05$ was considered to indicate a statistically significant difference.

\section{Results}

Patient characteristics. Among the consecutive 1,542 NSCLC patients, 729 patients had pathologically and/or cytologically confirmed NSCLC with distant metastasis. The characteristics of these patients are summarized in Table I. There were $509(69.8 \%)$ men and 220 women, with a median age of 69 years (range, 21-96 years). Among the patients, 250 (34.3\%), 234 (32.1\%), 207 (28.4\%), 122 (16.7\%), 98 (13.4\%) and 69 (9.5\%) had bone, lung, brain, adrenal gland, liver and extrathoracic lymph node metastases, respectively, whereas 283 (38.8\%) patients presented with pleural and/or pericardial fluid effusion.

Significance of each organ metastasis for survival. In the univariate analysis, patients with metastasis to the bone $(\mathrm{P}=0.024)$, adrenal glands $(\mathrm{P}<0.001)$, liver $(\mathrm{P}<0.001)$ and extrathoracic lymph nodes $(\mathrm{P}=0.014)$ exhibited a shorter survival compared to those without metastases to these sites. In a multivariate analysis using the Cox proportional hazards model, the presence of metastasis to the adrenal glands and the liver was associated with a poorer survival $(\mathrm{P}<0.001)$ (Table II).

Significance of metastasis to different body regions for survival. In the univariate analysis, patients with metastases to the brain/head $(\mathrm{P}=0.046)$, abdomen $(\mathrm{P}<0.001)$ and extremities $(\mathrm{P}=0.024)$ exhibited a poorer survival compared to those without metastases to these regions. However, metastasis to the thorax was not found to be an unfavorable prognostic factor $(P=0.226)$. In a multivariate analysis using the Cox proportional hazards model, only the presence of metastasis to the abdomen was associated with a poorer survival $(\mathrm{P}<0.001)$, whereas the presence of metastases to the brain/head $(\mathrm{P}=0.248)$ and/or the extremities $(\mathrm{P}=0.140)$ were not identified as unfavorable prognostic factors (Table III).

Association between metastatic region and PS. Using logistic regression analysis, metastases to the adrenal glands and pleural and/or pericardial fluid effusion were correlated with a poor PS of 2-4 (Table IV). As regards metastatic location, the presence of abdominal metastasis was found to be associated with a poor PS. However, no association was identified between specific organ metastases and response to chemotherapy in the logistic regression analysis (Table V).
Table I. Characteristics of 729 patients with metastatic non-small-cell lung cancer.

\begin{tabular}{|c|c|}
\hline Characteristics & No. $(\%)$ \\
\hline \multicolumn{2}{|l|}{ Age (years) } \\
\hline Median & 69 \\
\hline Range & $21-96$ \\
\hline \multicolumn{2}{|l|}{ Gender } \\
\hline Male & $509(69.8 \%)$ \\
\hline Female & $220(30.2 \%)$ \\
\hline \multicolumn{2}{|l|}{ Smoking history } \\
\hline Never & $192(26.3 \%)$ \\
\hline Current or former & $537(73.7 \%)$ \\
\hline \multicolumn{2}{|l|}{ ECOG PS } \\
\hline $0-1$ & $409(56.1 \%)$ \\
\hline $2-4$ & $320(43.9 \%)$ \\
\hline \multicolumn{2}{|l|}{ Histology } \\
\hline Adenocarcinoma & $543(74.5 \%)$ \\
\hline Squamous & $162(22.2 \%)$ \\
\hline Large-cell & $19(2.6 \%)$ \\
\hline Other & $5(0.7 \%)$ \\
\hline \multicolumn{2}{|l|}{ Treatment } \\
\hline Operation & $14(1.9 \%)$ \\
\hline Radiotherapy & $12(1.6 \%)$ \\
\hline Chemotherapy, CRT & $456(62.6 \%)$ \\
\hline Best supportive care & $247(33.9 \%)$ \\
\hline \multicolumn{2}{|l|}{ Metastatic site } \\
\hline Pleural/pericardial fluid & $283(38.8 \%)$ \\
\hline Bone & $250(34.3 \%)$ \\
\hline Lungs & $234(32.1 \%)$ \\
\hline Brain & $207(28.4 \%)$ \\
\hline Adrenal glands & $122(16.7 \%)$ \\
\hline Liver & $98(13.4 \%)$ \\
\hline Extrathoracic lymph nodes & $69(9.5 \%)$ \\
\hline Pleura & $41(5.6 \%)$ \\
\hline Other ${ }^{a}$ & $40(5.5 \%)$ \\
\hline
\end{tabular}

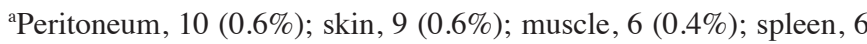
$(0.4 \%)$; pancreas, $3(0.2 \%)$; kidney, $3(0.2 \%)$; bone marrow, $2(0.1 \%)$; stomach, $2(0.1 \%)$; duodenum, $1(0.1 \%)$; jejunum/ileum, $1(0.1 \%)$; colon, 1 (0.1\%); nasal cavity, $1(0.1 \%)$; gingiva, $1(0.1 \%)$; retina, 1 $(0.1 \%)$; heart, $1(0.1 \%)$. ECOG, Eastern Cooperative Oncology Group; PS, performance status; CRT, chemoradiotherapy.

\section{Discussion}

In previous studies, the site of involvement did not appear to affect survival (10-12). However, other researchers reported that metastasis to specific organs may be associated with a poor prognosis (3-6). Finkelstein et al (3) reported that bone and liver metastases were identified as independent prognostic factors in 893 metastatic NSCLC patients. Sorensen et al (4) also demonstrated that NSCLC patients with brain metastasis exhibited a shorter survival compared to those without brain 
Table II. Survival analysis by organ/site.

A, Univariate analysis

Median OS (months)

\begin{tabular}{lccr} 
Organ/site & With metastasis & Without metastasis & P-value \\
\hline Pleural/pericardial fluid & 8.4 & 7.4 & 0.389 \\
Bone & 6.2 & 8.5 & 0.024 \\
Lungs & 6.9 & 7.9 & 0.469 \\
Brain & 6.7 & 8.3 & 0.071 \\
Adrenal glands & 3.9 & 8.9 & $<0.001$ \\
Liver & 3.8 & 8.7 & $<0.001$ \\
Extrathoracic lymph nodes & 5.8 & 8.0 & 0.014 \\
Pleura & 6.1 & 7.9 & 0.887 \\
Other & 4.9 & 8.0 & 0.020
\end{tabular}

$\mathrm{B}$, Multivariate analysis

\begin{tabular}{lccc}
\hline Organ/site & Hazard ratio & $95 \%$ CI & P-value \\
\hline Bone & 1.16 & $0.97-1.38$ & 0.102 \\
Adrenal glands & 1.83 & $1.47-2.28$ & $<0.001$ \\
Liver & 1.55 & $1.22-1.96$ & $<0.001$ \\
Extrathoracic lymph nodes & 1.31 & $0.99-1.73$ & 0.056 \\
\hline
\end{tabular}

OS, overall survival; CI, confidence interval.

Table III. Survival analysis by metastatic region.

A, Univariate analysis

Median OS (months)

\begin{tabular}{lccc} 
Body region & With metastasis & Without metastasis & P-value \\
\hline Brain/head & 6.6 & 8.4 & 0.046 \\
Thorax & 8.4 & 7.1 & 0.226 \\
Abdomen & 4.5 & 9.8 & $<0.001$ \\
Extremities & 6.1 & 8.5 & 0.024 \\
\hline
\end{tabular}

$\mathrm{B}$, Multivariate analysis

\begin{tabular}{lccc}
\hline Body region & Hazard ratio & $95 \%$ CI & P-value \\
\hline Brain/head & 1.11 & $0.93-1.34$ & 0.248 \\
Abdomen & 1.74 & $1.44-2.09$ & $<0.001$ \\
Extremities & 1.14 & $0.96-1.36$ & 0.140 \\
\hline
\end{tabular}

OS, overall survival; CI, confidence interval.

metastasis. In addition, Hoang et al (5) reported that skin and liver metastases were unfavorable prognostic factors in 1,436 patients with locally advanced or metastatic NSCLC. Recently, Bauml et al (6) reported that, among 376 NSCLC patients treated with systemic therapy, those with bone metastasis had a poor prognosis.

In the present study, the incidence of distant metastasis at the time of initial diagnosis of NSCLC was $47.3 \%$ and the most 
Table IV. Logistic regression analysis for factors associated with a poor performance status (PS) (2-4).

\begin{tabular}{lccc}
\hline Variables & Hazard ratio & 95\% CI & \\
\hline Metastatic organ & & $1.09-2.09$ & 0.013 \\
Pleural/pericardial fluid & 1.51 & $0.82-1.56$ & 0.456 \\
Bone & 1.13 & $0.83-1.58$ & 0.420 \\
Lungs & 1.14 & $0.73-1.46$ & 0.878 \\
Brain & 1.03 & $1.47-3.36$ & $<0.001$ \\
Adrenal glands & 2.23 & $0.90-2.20$ & 0.132 \\
Liver & 1.41 & $0.65-1.83$ & 0.745 \\
Extrathoracic lymph nodes & 1.09 & $0.51-1.87$ & 0.935 \\
Pleura & 0.97 & $0.73-1.45$ & \\
Metastatic region & & $0.99-1.92$ & 0.875 \\
Brain/head & 1.03 & $1.29-2.52$ & 0.059 \\
Thorax & 1.38 & $0.84-1.58$ & 0.396 \\
Abdomen & 1.80 & 1.15 & \\
Extremities & & & \\
\hline
\end{tabular}

CI, confidence interval.

Table V. Logistic regression analysis for factors associated with an unfavorable response to chemotherapy (SD + PD).

\begin{tabular}{lccc}
\hline Variables & Hazard ratio & 95\% CI & \\
\hline Metastatic organ & & & \\
Pleural and/or pericardial fluid & 0.84 & $0.53-1.32$ & 0.441 \\
Bone & 1.00 & $0.64-1.56$ & 0.994 \\
Lungs & 1.43 & $0.90-2.29$ & 0.130 \\
Brain & 1.31 & $0.78-2.19$ & 0.301 \\
Adrenal glands & 1.15 & $0.61-2.15$ & 0.671 \\
Liver & 1.32 & $0.68-2.59$ & 0.412 \\
Extrathoracic lymph nodes & 0.81 & $0.81-0.42$ & 0.528 \\
Pleura & 0.60 & $0.24-1.50$ & 0.271 \\
Metastatic region & & $0.89-2.47$ & $0.69-1.72$ \\
Brain/head & 1.48 & $0.61-1.61$ & $0.716-1.69$ \\
Thorax & 1.09 & 0.978 \\
Abdomen & 0.99 & 0.706 \\
Extremities & 1.09 & 0.716 \\
\hline
\end{tabular}

CI, confidence interval; SD, stable disease; $\mathrm{PD}$, progressive disease.

common metastatic sites were bone, lungs, brain, adrenal glands and liver. We investigated whether specific organ metastasis at presentation was of prognostic significance in NSCLC patients. In the multivariate analysis, the adrenal gland and liver metastases were identified as unfavorable prognostic factors. In the logistic regression analysis, adrenal gland metastasis and pleural and/or pericardial fluid effusion exhibited a statistically significant association with poor PS. When we evaluated metastasis to the head and neck, thorax, abdomen and extremities, only metastasis to abdominal organs was found to be an unfavorable prognostic factor in the uni- and multivariate analyses. In our logistic regression analysis, metastases to abdominal organs exhibited a statistically significant association with poor PS.
No metastasis other than abdominal was found to be associated with an unfavorable PS.

The results of this study may have important implications in clinical practice. First, brain and bone metastases were not unfavorable prognostic factors in our study; however, several previous studies reported brain metastasis to be one of the unfavorable prognostic factors, as neurological symptoms due to metastasis may be irreversible $(4,13,14)$. Bone metastasis has also been considered to be associated with poor survival due to skeletal-related events, such as pathological fractures, spinal cord compression and hypercalcemia of malignancy (15). The improved survival of patients with brain metastasis may be attributed to the discovery of early metastatic lesions by brain 
MRI, the introduction of stereotactic radiation therapy and the introduction of of epidermal growth factor receptor tyrosine-kinase inhibitors for the treatment of NSCLC patients. Zoledronic acid may also explain the improved survival in patients with bone metastases (16).

Second, liver metastasis was an unfavorable prognostic factor in our study, which was consistent with previously reported results $(3,5)$. Liver metastatic lesions are rarely associated with severe symptoms, although the majority of NSCLC patients had multiple nodules morphologically (17). It is well known that the majority of NSCLC patients with liver metastasis do not respond well to chemotherapy $(18,19)$. NSCLC may cause biliary tract obstruction by metastasizing to the lymph nodes in the porta hepatis or the hepatic parenchyma. The administration of chemotherapy may be complicated by liver metastasis regarding the activation or metabolism of several cytotoxic agents commonly used in the treatment of NSCLC. There have been several patients with liver metastases who were unable to continue chemotherapy due to liver dysfunction (20).

Third, adrenal gland metastasis was one of the unfavorable prognostic factors and was associated with a poor PS in this study, although adrenal metastasis per se rarely presents with severe symptoms (21) and only few patients with adrenal gland metastasis eventually develop adrenal insufficiency (22). It has not been determined whether patients with adrenal gland metastasis also present with unfavorable several organ metastases or with unfavorable metastatic patterns. However, adrenal gland metastasis per se was found to be an unfavorable factor, even in the multivariate analysis. The precise etiology of adrenal metastasis being an unfavorable prognostic factor remain unclear. In the future, we aim to evaluate metastatic factors using a cluster analysis to elucidate the reasons underlying this finding. Further studies are required to confirm the effects of these organ metastases as observed in the present study.

Despite these significant findings, there were certain limitations to this study. First, the design of the study was retrospective and, therefore, complicated by lead time and length time biases. Second, there was lack of information regarding the development of distant metastases during the clinical course of the disease. However, despite these limitations, our findings may be of clinical value for the future management of NSCLC patients from unselected groups. Our results confirmed that the therapeutic approach to the treatment of NSCLC patients with distant metastases is complicated.

In summary, our results suggest that liver and adrenal gland metastases adversely affect the outcome of NSCLC, whereas brain and bone metastases do not. Therefore, when deciding on the administration of agressive therapy, which may increase treatment-related mortality, the individual patient's medical condition, including coexistence of such metastases, should be taken into consideration.

\section{References}

1. Matsuda A, Matsuda T, Shibata A, Katanoda K, Sobue T and Nishimoto H; Japan Cancer Surveillance Research Group: Cancer incidence and incidence rates in Japan in 2008: a study of 25 population-based cancer registries for the Monitoring of Cancer Incidence in Japan (MCIJ) project. Jpn J Clin Oncol 44: 388-396, 2014.
2. Little AG, Gay EG, Gaspar LE and Stewart AK: National survey of non-small cell lung cancer in the United States: epidemiology, pathology and patterns of care. Lung Cancer 57: 253-260, 2007.

3. Finkelstein DM, Ettinger DS and Ruckdeschel JC: Long-term survivors in metastatic non-small-cell lung cancer: an Eastern Cooperative Oncology Group Study. J Clin Oncol 4: 702-709, 1986.

4. Sorensen JB, Hansen HH, Hansen M and Dombernowsky P: Brain metastases in adenocarcinoma of the lung: frequency, risk groups, and prognosis. J Clin Oncol 6: 1474-1480, 1988.

5. Hoang T, Xu R, Schiller JH, Bonomi P and Johnson DH: Clinical model to predict survival in chemonaive patients with advanced non-small-cell lung cancer treated with third-generation chemotherapy regimens based on Eastern Cooperative Oncology Group data. J Clin Oncol 23: 175-183, 2005.

6. Bauml J, Mick R, Zhang Y, Watt CD, Vachani A, Aggarwal C, Evans T and Langer C: Determinants of survival in advanced non-small-cell lung cancer in the era of targeted therapies. Clin Lung Cancer 14: 581-591, 2013.

7. Travis WD, Brambilla E, Muller-Hermelink HK and Harris CC (eds): Pathology and genetics of tumours of the lung, pleura, thymus and heart. In: World Health Organization Classification of Tumours. IARC Press, Lyon, pp9-124, 2004.

8. Sobin LH, Gospodarowicz MK and Wittekind C (eds): TNM Classification of Malignant Tumours. 7th edition. Wiley-Blackwell, pp136-146, 2009.

9. Cox DR: Regression models and life tables. J Roy Statist Soc Ser B Metho 34: 187-220, 1972.

10. Sakurai M, Shinkai T, Eguchi K, et al: Prognostic factors in non-small cell lung cancer: multiregression analysis in the National Cancer Center Hospital (Japan). J Cancer Res Clin Oncol 113: 563-566, 1987.

11. Borges M, Sculier JP, Paesmans M, et al: Prognostic factors for response to chemotherapy containing platinum derivatives in patients with unresectable non-small cell lung cancer (NSCLC). Lung Cancer 16: 21-33, 1996.

12. Paralkar VR, Li T and Langer CJ: Population characteristics and prognostic factors in metastatic non-small-cell lung cancer: a Fox Chase Cancer Center retrospective. Clin Lung Cancer 9: 116-121, 2008.

13. Jacot W, Quantin X, Boher JM, Andre F, Moreau L, Gainet M, Depierre A, Quoix E, Chevalier TL and Pujol JL; Association d'Enseignement et de Recherche des Internes en Oncologie: Brain metastases at the time of presentation of non-small cell lung cancer: a multi-centric AERIO analysis of prognostic factors. Br J Cancer 84: 903-909, 2001.

14. Sanchez de Cos J, Sojo Gonzalez MA, Montero MV, Perez Calvo MC, Vicente MJ and Valle MH: Non-small cell lung cancer and silent brain metastasis. Survival and prognostic factors. Lung Cancer 63: 140-145, 2009.

15. Saad F, Lipton A, Cook R, Chen YM, Smith M and Coleman R: Pathologic fractures correlate with reduced survival in patients with malignant bone disease. Cancer 110: 1860-1867, 2007.

16. Zarogoulidis K, Boutsikou E, Zarogoulidis P, Eleftheriadou E, Kontakiotis T, Lithoxopoulou H, Tzanakakis G, Kanakis I and Karamanos NK: The impact of zoledronic acid therapy in survival of lung cancer patients with bone metastasis. Int J Cancer 125: 1705-1709, 2009.

17. Kagohashi K, Satoh H, Ishikawa H, Ohtsuka M and Sekizawa K: Liver metastasis at the time of initial diagnosis of lung cancer. Med Oncol 20: 25-28, 2003.

18. Gorg C, Schwerk WB, Wolf M and Havemann K: Prognostic value of response to chemotherapy using ultrasound in lung cancer with metastatic liver involvement. Bildgebung 57: 70-73, 1990.

19. Yamamoto N, Tamura T, Fukuoka M and Saijo N: Survival and prognostic factors in lung cancer patients treated in phase I trials: Japanese experience. Int J Oncol 15: 737-741, 1999.

20. Nakagawa T, Okumura N, Ohata K, Igai H, Matsuoka T and Kameyama K: Postrecurrence survival in patients with stage I non-small cell lung cancer. Eur J Cardiothorac Surg 34: 499-504, 2008.

21. Toloza EM, Harpole L and McCrory DC: Noninvasive staging of non-small cell lung cancer: a review of the current evidence. Chest 123 (Suppl 1): 137S-146S, 2003.

22. Mohammad K and Sadikot RT: Adrenal insufficiency as a presenting manifestation of nonsmall cell lung cancer. South Med J 102: 665-667, 2009. 